\title{
EXPERIMENTAL ANALYSIS OF BULK MATERIAL FLOW THROUGH HOPPER
}

\author{
Ivo Vaicis, Kristaps Spade, Alexander Janushevskis, Alexander Boiko \\ Riga Technical University, Latvia \\ ivo.vaicis@rtu.lv
}

\begin{abstract}
Hoppers are used in various fields of application, as for material transportation in food industry, mining, agricultural industry, transport industry etc. Mass flow rate at the hopper outlet nozzle is one of the significant parameters which is used to evaluate efficiency of the hopper. During the discharge process of hopper, different effects in bulk material behavior, as accumulation or bridging of particles, could occur, therefore decreasing efficiency of the hopper discharge rate. In this paper physical and numerical experiments of bulk flow through the hopper are performed and analyzed under applied vertical vibrations to the hopper construction. Vibrations are considered with different frequency and amplitude in experimental and numerical models. As the bulk material spherical glass particles are used in the experiments. The obtained results of the physical experiments are compared with the results of numerical simulations, therefore allowing to evaluate efficiency of numerical models used for analysis of the bulk material flow effects in the hopper system. The obtained experimental and numerical model results will be used for further analysis of a more complicated hopper model, where the bulk material segregation process at the outlet nozzle of the hopper system and dust dispersion in surrounding environment during the bulk material loading process will be taken in account.
\end{abstract}

Keywords: dust control, numerical modeling, bulk material, metamodeling.

\section{Introduction}

Hopper systems are widely used in different fields of application - for storing and transporting granular materials, in mining, agriculture, wood chip production etc. Dependent on the granular medium and the hopper shape properties, different flow types - arching, funnel flow, flow with particle segregation and other may occur during the hopper discharge process (Fig. 1) [1].

a)

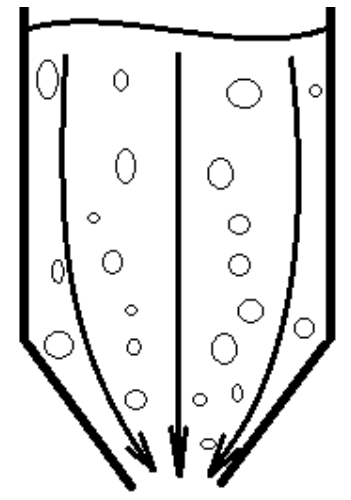

b)

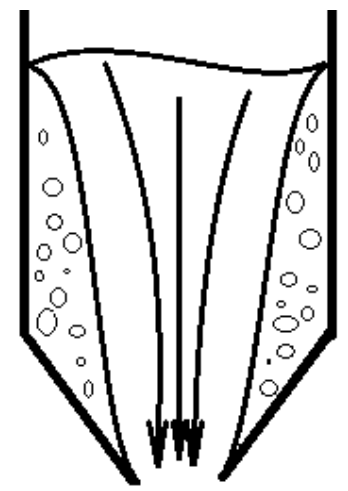

Fig. 1. Granular medium flow types: $a$ - mass flow; $b$ - funnel flow

The hopper discharge angle has significant importance on the granular material discharge rate. Mathematical models for design of appropriate hoper discharge angle have been developed [2]. By using the Jenike's theory, it is possible to find the appropriate hopper discharge angle to obtain mass flow [1]. Different analytical approaches are used to analyse the hopper mass discharge, for example, Carleton and Williams methods, which allow to determinate the mass flow rate for a hopper with the angle of internal friction in the range $20-50^{\circ}$ [3]. In practice there are limitations to do changes in the hopper design and change the flow type. Applied vibrations are often used to prevent the hopper system from jamming and improve the hopper discharge rate [4]. There are different parameters (granular material shape, size, density, rigidity and others), which should be considered when appropriate vibration regime is selected for a specific hopper system. Prediction of the bulk material behaviour in the hopper system involves testing which is time and cost consuming method. Development of computational methods allows to use DEM (Discrete Element Method) in combination with CFD (Computational Fluid Dynamics) to simulate the bulk material behaviour and interaction with geometries at different boundary conditions, therefore allowing to save time used for testing. In this paper the discharge rate of the hopper system is analysed and compared with physical experiments and numerical models. 


\section{Setup of numerical and physical experiments}

Vibrations are often implemented in hopper systems to prevent the hopper from jamming. If the hopper is discharging without applied vibrations, in many cases the obtained flow type is funnel flow. When horizontal vibrations are applied on the hopper system, the flow state changes into inverted funnel flow where material along the hopper side exits first. Applied vibration in horizontal direction increases also the hopper discharge rate [5]. In a hopper with vertical applied vibrations the discharge rate is decreasing with respect to acceleration (1) [6].

$$
\Gamma=\frac{a \omega^{2}}{g},
$$

where $\Gamma$-dimensionless acceleration;

$a$ - amplitude of vibration, m;

$g$ - gravity acceleration, $\mathrm{m} \cdot \mathrm{s}^{-2}$.

In this paper analysis of the hopper discharge rate is performed with applied vertical vibrations in the range $\Gamma=0-5$, where the frequency range $f=0-35 \mathrm{~Hz}$. For physical and numerical experiments (Fig. 2, b) spherical glass particles with diameter 1.0-1.3 mm are used. The hopper construction is made from plastics with the mechanical properties listed in Table 1.

Physical experiments (Fig. 2, a) are performed with a hopper mounted on vibration exciter. Hopper is filled with the glass particles and measurements of the discharge process are performed at different vibration frequency of exciter while the amplitude of vibrations is constant. The discharge process is captured using video recording with 300 frames per second.

Discharge rate is analysed changing vibration acceleration by the change of frequency. In numerical model Hertz - Mindlin contact model is used for particle - particle and particle - geometry contact [7]. Euler time integration method with fixed time step $3.6 \cdot 10^{-6} \mathrm{~s}$ is used for simulation of the discharge process.

a)

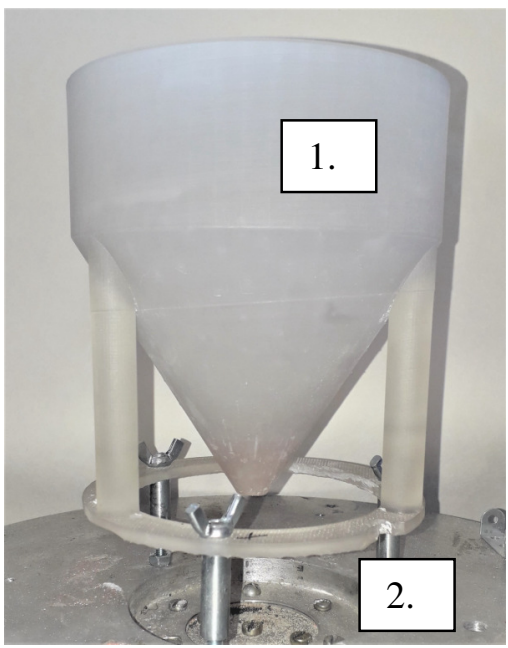

b)

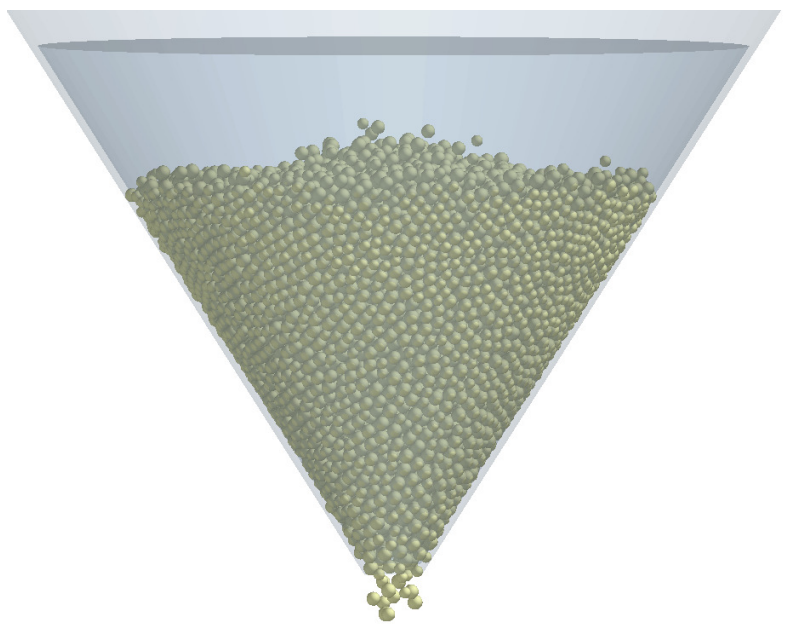

Fig. 2. Experimental setup: a - physical model (1 - hopper, 2 - vibration exciter); $\mathrm{b}$ - numerical model in EDEM [8]

Table 1

Mechanical properties

\begin{tabular}{|c|c|c|}
\hline Mechanical properties & Glass particles & Hopper construction \\
\hline Density $\left(\mathrm{kg} \cdot \mathrm{m}^{-3}\right)$ & 2500 & 1700 \\
\hline Coefficient of static friction & 0.1 & 0.5 \\
\hline Poisson's ratio & 0.25 & 0.32 \\
\hline Coefficient of restitution & 0.8 & 0.6 \\
\hline
\end{tabular}




\section{Results and discussion}

Physical and numerical experiments are performed for the hopper system with applied vertical vibrations and analysis of dimensionless mass discharge (ratio between the discharge rate with applied vibration and discharge rate without applied vibrations). In the numerical model the theoretical value of the friction coefficient for the hopper is used and air resistance is neglected. Physical and numerical experiments are indicating that the hopper discharge rate is decreasing with increase of acceleration (Fig. 3.). The obtained results comply with other researches performed by Hunt L. et al. [6].

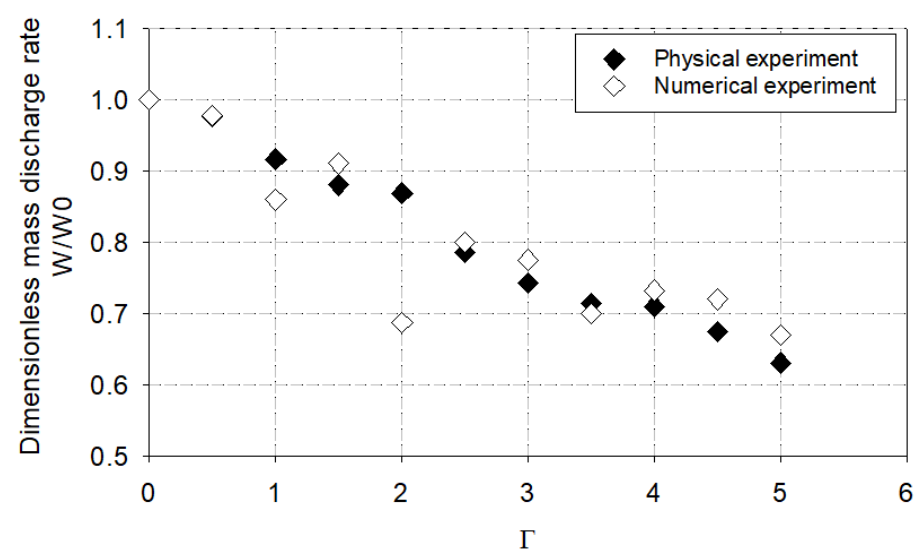

Fig. 3. Dimensionless mass discharge rate in numerical and physical experiments

Results of the numerical experiment show that the discharge rate is significantly dependent on the applied vertical vibrations. Second order polynomial regression [9] is used to approximate the results of the physical experiments (Fig. 4, a). The used approximation has the mean square cross validation error $\sigma^{2}=0.0197$ and $R^{2}=0.98(2)$, indicating that the used approximation has sufficient accuracy.
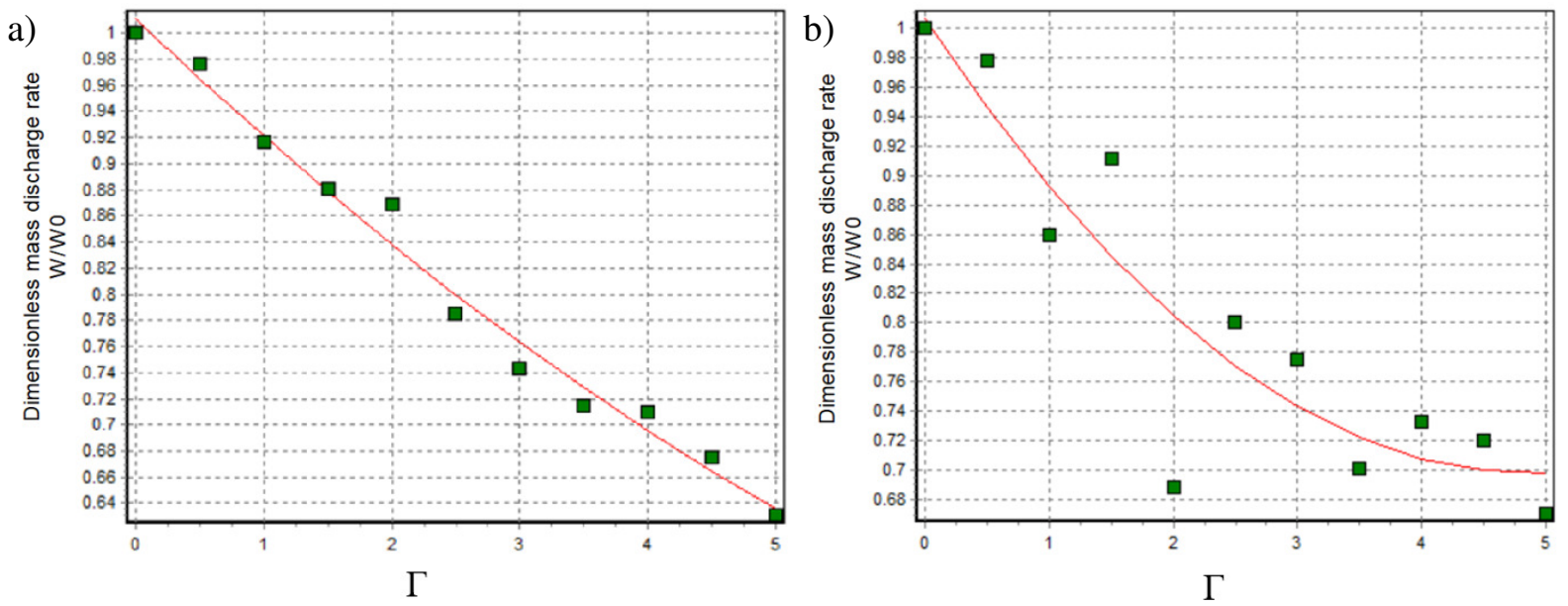

Fig. 4. Second order polynomial approximation of results - a) physical experiment, b) numerical experiment

Approximation of the numerical results (Fig. 4, b) has less accuracy with $\sigma^{2}=0.061$ and $R^{2}=0.78$. By eliminating bad point (experiment point -5 ), accuracy of approximation has significant increase where the mean square cross validation error $\sigma^{2}=0.035$ and $R^{2}=0.92$.

$$
R^{2}=1-\left(\frac{\sigma_{\text {test }- \text { rel }}}{100}\right)^{2}
$$

where $\sigma_{\text {test rel }}-$ relative approximation prediction error.

The results indicate that during the deceleration phase of the vibration cycle particles loose contact with the hopper wall and fall under gravity. With increase of vibration acceleration increases also the discharge amplitude, but the average discharge rate is decreasing (Fig. 5). 


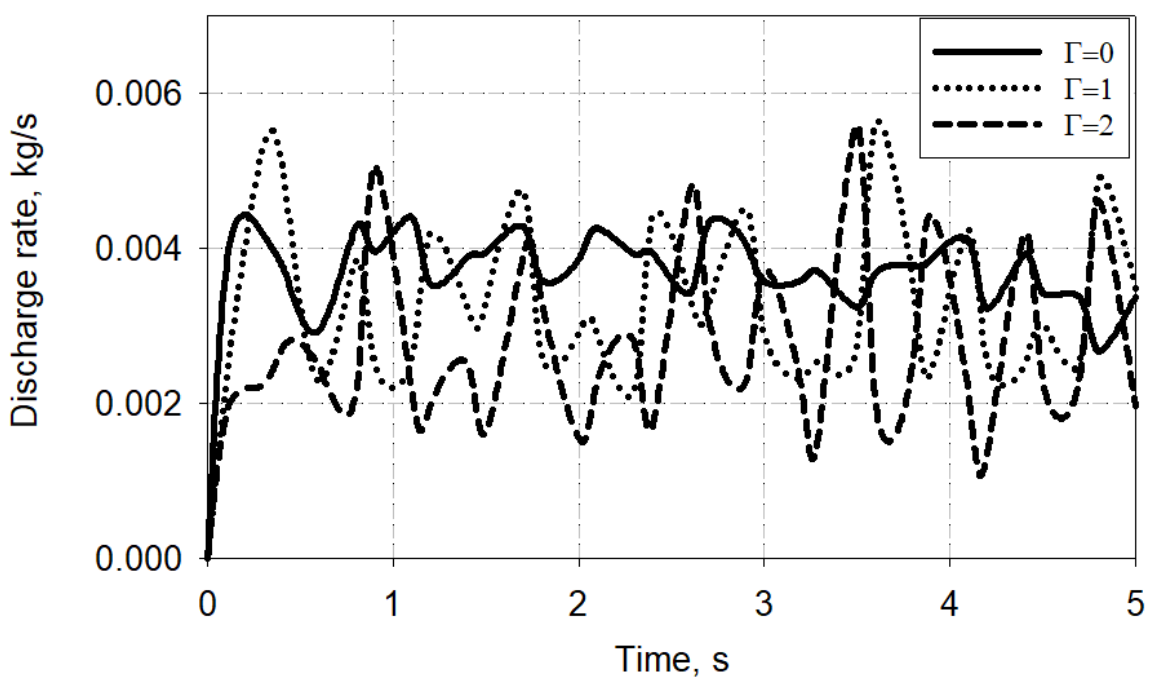

Fig. 5. Discharge rate dependence on acceleration $\Gamma$

A high friction coefficient of the hopper wall may cause particle accumulation in the hopper, therefore, reducing discharge rate and material jamming can occur [10]. Different methods in literature are used to analyse flow types in a hopper as analytic approaches, which allow to determine the flow type and complex physical experiments involving X-ray tomography [11].

In the performed numerical analysis the particle velocity field is analysed. In the model without applied vibrations the mass flow state is obtained where all particles uniformly tend to exit the hopper (Fig. 5, a). In the performed experiments a hopper with a small outlet nozzle diameter is used (3). In hoppers with small outlet nozzle diameters granular material is developing mass flow type [12].

$$
D=5 d,
$$

where $D$-hopper outlet diameter, m;

$d$-diameter of particle, $\mathrm{m}$.

Applied vertical vibrations on the hopper may reduce the possibility of material jamming in the hopper and change material discharge type from funnel flow to mass flow [13]. During the acceleration phase of the vibration cycle, particles are losing contact with the hopper wall, therefore limiting the possibility to accumulate in the stagnation zone (Fig. 6, b).

a) velocity, $\mathrm{m} / \mathrm{s}$

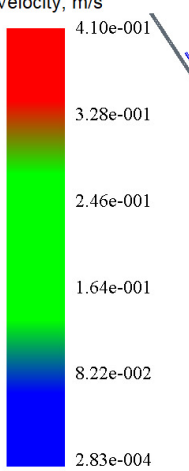

b)

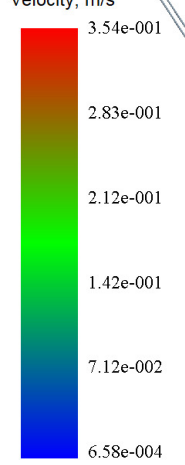

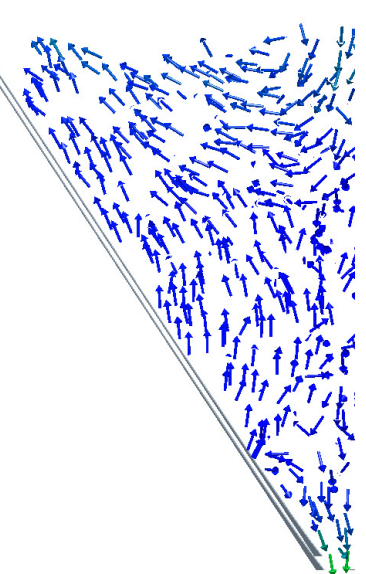

Fig. 6. Velocity field of particles at 2s from start of simulation: $\mathrm{a}$ - model without applied vibrations; $\mathrm{b}$ - model with applied vibrations

Another model is used to analyse the effect of the friction coefficient of the hopper construction on the granular material flow type. For silos with funnel flow material stagnation zones are observed close to the hopper walls [14]. In model with small friction coefficient $(\mu=0.2)$ the mass flow state is 
obtained (Fig. 6. a.) and with increase of the friction coefficient $(\mu=0.9)$ the mass flow changes into funnel flow, where particles in the flow centre tend to exit the hopper first, while material stagnation zones are developing close to the hopper wall (Fig. 7).

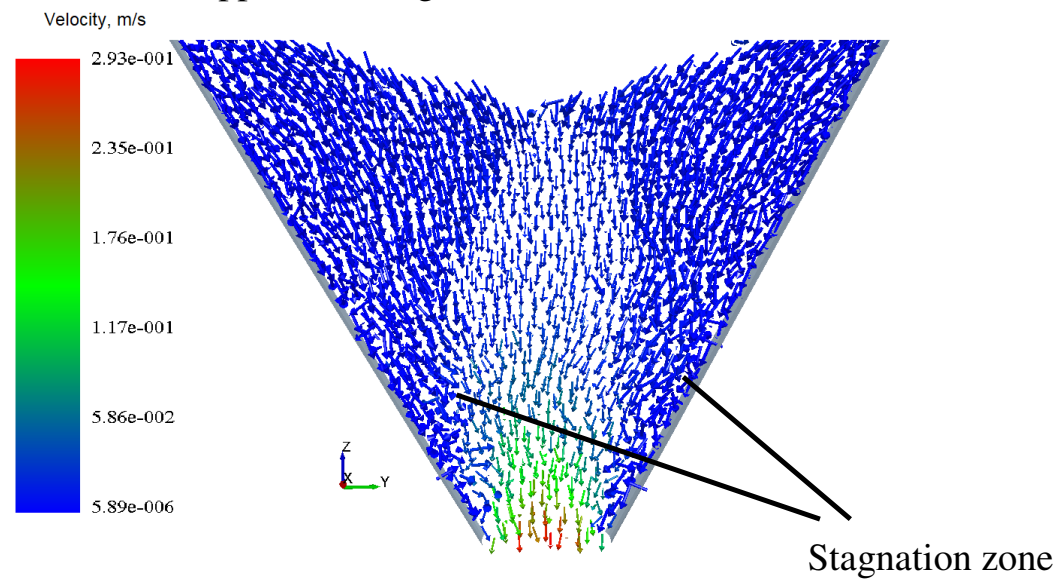

Fig. 7. Funnel flow - particle velocity distribution (friction coefficient $\boldsymbol{\mu}=\mathbf{0 . 9}$ )

In model with a high friction coefficient vertical vibrations with the frequency $10 \mathrm{~Hz}$ and amplitude $0.001 \mathrm{~m}$ are applied and results without material stagnation zones in the hopper are obtained. Velocity of particles is uniformly distributed during acceleration (Fig. 8, a) and deceleration (Fig. 8, b) stages of applied harmonic vibrations and particles are not sticking to the hopper wall, therefore the granular flow tends to approach the mass flow type.

a)

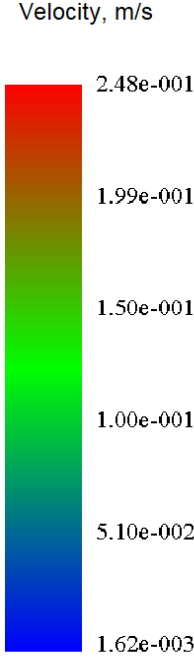

b)

Veloctiy, $\mathrm{m} / \mathrm{s}$

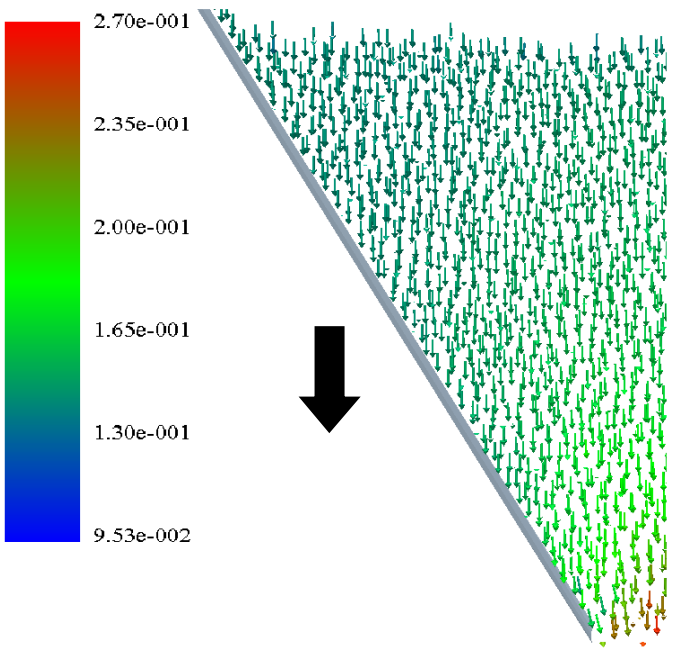

Fig. 8. Particle velocity distribution with applied vibrations $(f=10 \mathrm{~Hz}, A=0.001 \mathrm{~m})$ on hopper

(friction coefficient $\boldsymbol{\mu}=\mathbf{0 . 9}$ ): $\mathrm{a}$ - acceleration stage; $\mathrm{b}$ - deceleration stage

The created numerical model can be used for further metamodel based shape optimization of the hopper design parameters considering uncertainty due to the mechanical properties of various bulk materials [15].

\section{Conclusions}

1. The results of the performed physical and numerical experiments show that applied vertical vibrations on the hopper system have significant impact on the discharge rate. With increase of the vibration acceleration to $\Gamma=5$, the dimensionless discharge rate is decreasing by $37 \%$ comparing with the discharge rate for the hopper without applied vibrations.

2. The created numerical model can be used for analysis of the particle flow field to evaluate the granular material flow type in the hopper and also for evaluation of the hopper discharge rate. The numerical results have average difference within $5 \%$ from the results of the physical experiments. 
3. Applied vertical vibrations on the hopper reduce the wall friction impact on the granular flow type. In the hopper deceleration stage particles are losing contact with the hopper wall and are sucked into flow.

4. In further research a more exact numerical model should be used including effects of the air resistance and induced air flow due to the particle motion.

\section{Acknowledgements}

This research is funded by the Latvian Council of Science, project "Creation of design of experiments and metamodeling methods for optimization of dynamics of multibody 3D systems interacting with bulk solids and fluids", project No. Izp-2018/2-0281.

\section{References}

[1] Lobato J.C.M., Mesquita A. Conical hopper design for mass flow - case study for red mud powder. Proceedings of ENIT2014. 2014. pp. 120-131.

[2] Jenike W. A., Johanson R.J. Bin Loads. Journal of the Structural Division. Vol.94. 1968. pp. 1011-1042.

[3] Janda A., Maza D., Unjaming a granular hopper by vibration. Europhysics Letters. 2009. 24002 p1. -24002 p6.

[4] Woodcock C.R., Masson S.J. Bulk Solids Handling: An introduction to the Practice and Technology. Blackie Academic \& Professional. Glasgow. 1993. ISBN 978-94-010-7689-0, pp. 58-61.

[5] Hunt L. M., Weathers C.R. Effects of horizontal vibration on hopper flows of granular materials. Physics of Fluids. Vol. 11. 1999. pp. 68-75.

[6] Hunt L. M., Wassgren C. Effects of Vertical Vibration on Hopper Flows of Granular Material. Physics of Fluids. Vol. 14. 2002. pp. 335-348.

[7] Gelnar D., Zegzulka J. Discrete Element Method in the Design of Transport Systems. Verification and Validations of 3D models. Switzerland: 2019.

[8] EDEM 2019, Discrete Element Modeling Software, DEM Solution Ltd.

[9] Auzins J., Janusevkis A. Eksperimentu plānošana un analīze. RTU izdevniecība. Rīga, 2007. ISBN-978-9984-32-157-8, pp. 165-167.

[10]Zhang S., Lin P. Investigating the influence of wall frictions on hopper flows. Granular Matter. Springer Verlag. 2014. pp. 857-866.

[11] Grudzien K., Detection of Tracer Particles in Tomography Images for Analysis of Gravitational Flow in Silo. Image \& Communications. Vol. 18, 2013. pp. 11-22.

[12] Megalhaes F., Atman A., Analysis of the velocity field of granular hopper flow. Granular Matter. Springer Verlag. 2015.

[13] Arnold P.C., Kaaden A.S. Reducing hopper wall friction by mechanical vibration. Powder Technology. Vol. 16, 1977. pp. 63-66.

[14] Ristow H. G., Hermann J.H. Forces on the walls and stagnation zones in a hopper filled with granular material. Physica A: Statistical Mechanics and its Applications. Vol. 213, 1995. pp. 474481.

[15] Janushevskis A., Melnikovs A., Janusevskis J. Robust Shape Optimization of Composite Structure Using Metamodels. Engineering Optimization IV - Chapter 121, 2014, () 2015 Taylor \& Francis Group, London, ISBN 978-1-138-02725-1, pp. 715-720. 